\title{
Dotgain estimation using linear least squares incorporating neighboring and clustering effects
}

\author{
T. Stamm and K. Simon \\ Swiss Federal Laboratory for Materials Testing and Research (EMPA), Ueberlandstrasse 129, \\ 8600 Duebendorf, Switzerland
}

\begin{abstract}
This work presents a model for dotgain prediction using repetitive patterns based on the characterization of neighboring and clustering effects of a specific printing device. Estimating dotgain is done nowadays by measuring patches of color patterns realized by a specific printing device. Current models use the information about adjacent dots to predict dotgain. However, research has shown that dotgain is influenced by the neighborhood of a dot which in general is bigger than one dot-size, in particular in connection with laser printers. The presented method predicts the dotgain of a dot considering a larger surrounding based on the observation of two main parameters affecting the luminance of a pattern which can be fitted using linear regression.
\end{abstract}

Keywords: Dotgain prediction, cluster detection, linear least squares, pattern entropy

\section{INTRODUCTION}

Todays printing devices commonly rely on amplitude modulated (AM) or frequency modulated (FM) halftoning techniques to create the impression of continuous color tones. The modulation of the high resolution dots generally result in differences between the originally intended, theoretical ink coverage and the actual perception. In first order, the ink spread (known as "mechanical" or "physical" dotgain) introduces a deviation from an ideal dot in manifold and mostly unknown ways. In second order, light gets absorbed on a complex optical path within substrate and ink, resulting in "optical" dotgain ${ }^{1}$.

Dotgain describes a luminance change within the surrounding of a dot, accordingly, several models for predicting dotgain with incorporation of the surrounding exist, for example, the "circular dot overlap model" 7 which models the boundary overlappings of dots. However, recent works based on the adjacency effects described by Neugebauer ${ }^{4}$ have shown that the dotgain of one single dot can be noticeably affected by dots within a distance of several dot-sizes. A desirable model for dotgain could therefore be described by the measurement of representative patterns modeling larger surroundings of a dot.

Wang $^{2}$ considered the measurement of $2 \times 2$ patterns in order to predict larger dot constellations. These repetitive patterns are printed and measured using a densitometer. An arbitrary given bitmap of any size can then be subdivided into $2 \times 2$ cells and the resulting luminance value can therefore be predicted by a linear combination of the luminances of the measured patterns weighted with the number of occurencies of the corresponding $2 \times 2$ cells in the bitmap.

In this work, we present a new approach to predict complex dot patterns. Our approach is based on an extended analysis of repetitive patterns. We give a general overview of the measuring task in section 2 and discuss design ideas and observations made during experiments in section 3 . In section 4 and 5 , we describe a model based on the observations, whereas we will show that using only a few explicitly designed patterns, one can estimate dotgain with high accuracy. A comparison between our prediction concept and the method of Wang shows a significant improvement in accuracy which will be discussed in section 6 .

Further author information: tobias.stamm@empa.ch, +41 4482357 60, klaus.simon@empa.ch, +41 448234171

Color Imaging XIV: Displaying, Processing, Hardcopy, and Applications, edited by Reiner Eschbach Gabriel G. Marcu, Shoji Tominaga, Alessandro Rizzi, Proc. of SPIE-IS\&T Electronic Imaging SPIE Vol. 7241, 72411A · C 2009 SPIE-IS\&T · CCC code: 0277-786X/09/\$18 · doi: 10.1117/12.805302 


\section{MEASURING REPETITIVE PATTERNS}

In our test environment, monochromatic grey scale prints of a Xerox Phaser 7750 laser printer are considered. Like many others, this printer has the restriction to either print or not print dots at specific positions defined by a two-dimensional bit mask. The printer offers a maximal resolution of 1200 dpi which would have been preferable for testing reasons but an accurate placing of the dots at this resolution was not possible, therefore, a lower resolution of $600 \mathrm{dpi}$ was used for all prints. In this text, sometimes the term pixel is used which denotes a unit that has been chosen to match the desired resolution of the printer so that a black pixel is equal to a printed dot, whereas a white pixel corresponds to an unprinted dot.

This kind of printers have to use a bilevel halftoning algorithm to create the impression of grey levels. The main idea behind halftoning is to exploit the limited resolution of the human eye to create the illusion of a grey value by the seeming mixture of the luminances in the smallest perceivable area relative to the reading distance. The human eye resolves about 1 arc minute which corresponds to a field of view of about $0.12 \mathrm{~mm}$ at normal reading distance. ${ }^{5}$ In this work, we used an eyeOne-densitometer as a measuring device, which has a circular field of view of about $4.7 \mathrm{~mm}$ in diameter ${ }^{6}$ and therefore averages the luminances captured within a much bigger area than the human eye.

To compare the theoretical coverage of the originally intended bit mask with its realization by the printer, we have to account for all dots within the field of view. The large field of view of the eyeOne device in general makes it impractical to account for all dots. But when the halftoning consists of regularly repetitive cells (Figure 1a), we can assume the overall coverage within the field of view (Figure 1b) to be approximately the same as the coverage of one single cell. The goal of this work is to estimate the luminance of a dot including its surrounding by looking at regular patterns tiled by repetition of quadratic $k \times k$ cells where $k$ stands for the number of pixels in one dimension.

As described in the paper of Rasmussen ${ }^{8}$, laser printers produce unsteady printing results. We verified this by printing the $3 \times 3$ pattern shown in figure 1a over the whole page with $600 \mathrm{dpi}$. In figure 1c, one can clearly see streaks and a non-uniformity in lightness over the whole page. The contrast of figure 1c has been amplified for visualization reasons but also in reality, a difference of up to $10 \Delta L$ between luminance values at different positions on the paper were measured. In this paper, we denote luminance values as the $L^{*}$-value of the CIELAB color space which is commonly used as a space of linear perception and $\Delta L$ denotes the difference of two $L^{*}$ values. To get reliable luminance results, all patches in this work have been printed several times at different positions on the paper. The results have been statistically averaged.

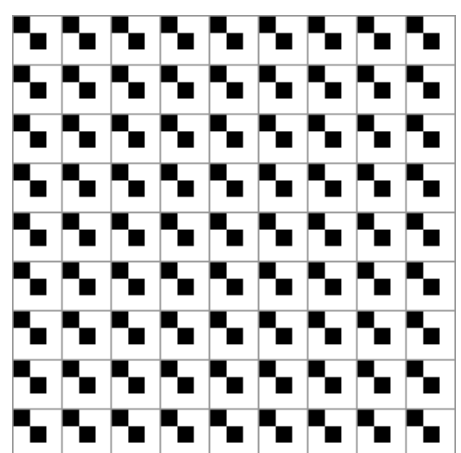

(a) $3 \times 3$ pattern

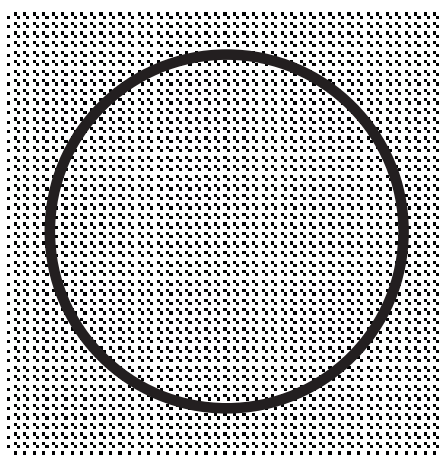

(b) field of view of the eyeOne

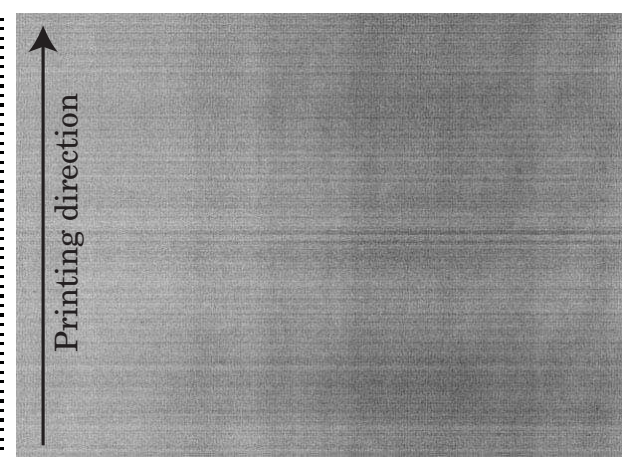

(c) Scan of a printed A4-page

Figure 1. Repetitive patterns: Simulation of a grey value which in real printers may show strong irregularities.

When measuring a patch with a repetitive pattern with the eyeOne device, an exact positioning is not practical which means that patterns that are equal to others by translation are considered to be equivalent. In the following text, only distinct patterns will be used. 


\section{OBSERVATIONS}

When we assume the dotgain to influence the luminance of dots up to a distance of about 2 dots, the quadratic surrounding of a middle dot defines a $5 \times 5$ cell. Every possible arrangement of dots within such a cell can be represented by a binary mask $M$ depicting where to set a black or white pixel. There are $2^{5 \times 5} \approx 3.36 \cdot 10^{7}$ possible arrangements for a $5 \times 5$ cell. This shows that even for surroundings of only a few dots, the number of representative patches grows exponentially, therefore a measurement of all arrangements of large cells is impractical. On the other hand, this knowledge is of great interest in the context of halftoning algorithms. For that reason, larger cells are usually simulated or predicted. Thereby, the goal is to reduce the necessary measurements to a handful of patterns.

The theoretical coverage $c$ of $M$ means the number of printed black dots divided by the maximal number of black dots $(k \times k)$ within a cell. Additionally, the surface $S$ of $M$ stands for the number of boundaries between printed and unprinted pixels. Since dotgain is known to occur mostly in the boundary regions of printed areas, cells with a low $S$ are assumed to show less dotgain than cells with large $S$. Since the AM rasters used in todays printing devices (dot, line-screen, chain- $\operatorname{dot}^{10}$ ), by definition, have less surface than FM rasters, this corresponds directly to the observation that halftoning using AM in general shows less dotgain than FM.

We suppose the values $c$ and $S$ of all arrangements of a $5 \times 5$ cell are determined. By exhaustive search, we observe minimal and maximal $S$ for every $c$, resulting in 148 distinct patterns showing a minimal surface $S_{\text {min }}$ and 2152 distinct patterns showing a maximal surface $S_{\text {max }}$. These resulting 2300 patches have been printed and measured. The measured luminances of these patterns can be observed in figure 2, where the grey points represent the patterns with minimal $S$ and the black points the patterns with maximal $S$. The circles represent minimal and maximal luminances for every $c$ whereas the lines in between the circles indicate the spread of the observed luminance values.

It is common to study extremal instances both in combinatoric and in system calibration. Therefore, for every $c$, the arrangements with the minimal and maximal measured luminance $L_{\min }$ and $L_{\max }$ both of the patterns with minimal and maximal surface $S_{\min }$ and $S_{\max }$ have been identified, resulting in the 104 patterns shown in figure 3. As mentioned above, the patterns with maximal $S$ are correlated to FM-like patterns whereas the patterns with minimal $S$ show some kind of an AM-like line screen.

In the following, we are interested in the differences between the patterns resulting in minimal and maximal luminances. We observe that the patterns with maximal $S$ tend to either form clusters or diffuse the dots as good as possible resulting in a difference between $L_{\min }$ and $L_{\max }$ of up to $6 \Delta L$. This observation will play a major role in section 4 , where we define a model for such clusters. In the dark regions, the clustered patterns contribute to the $L_{\max }$ patterns, whereas in the light regions the clusters contribute to the $L_{\min }$ patterns. Therefore, to describe a dotgain model incorporating clusters with a linear model, the theoretical ink coverage $c$ must be decomposed into independent parts which we will discuss in section 5 . We note that we also observe a directional dependency but we neglect the luminance differences for now and save this observation for future work. 


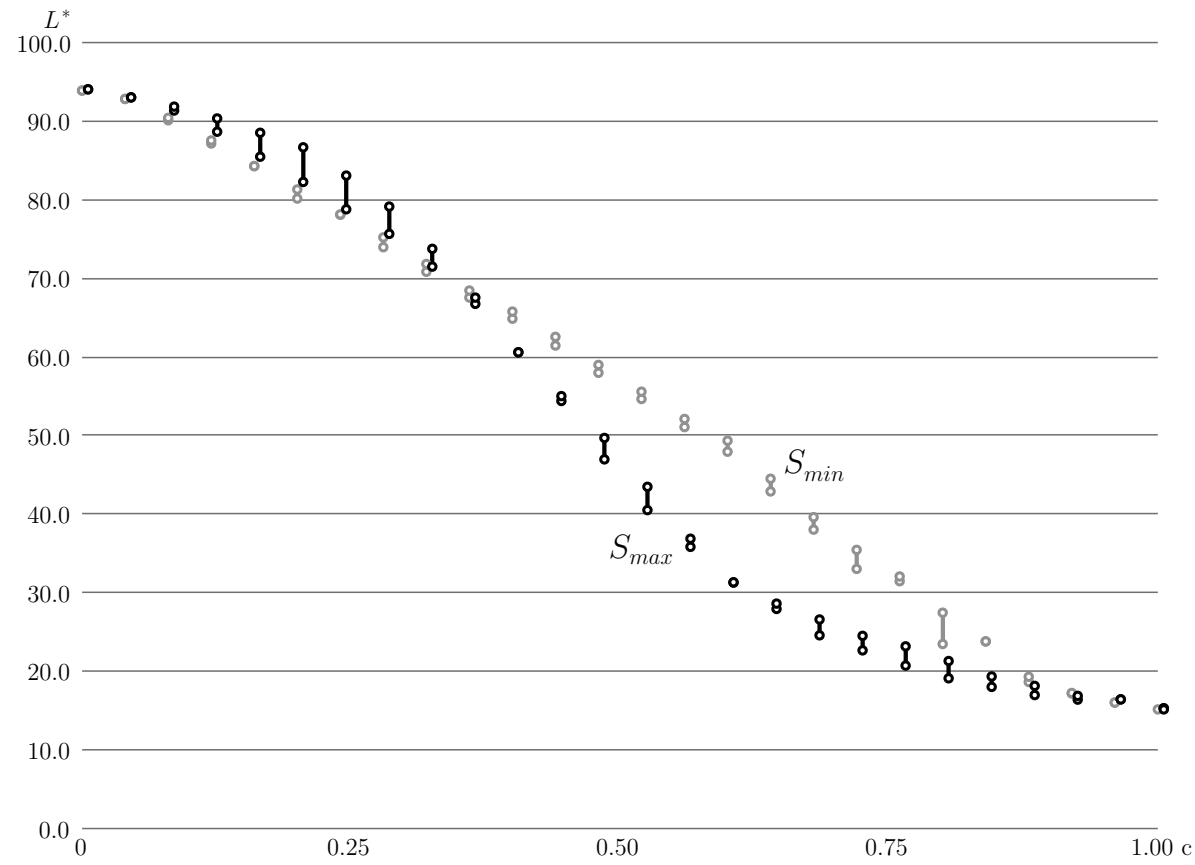

Figure 2. The luminances of the patches of figure 3. Black dots depict the $S_{\text {max }}$ patches whereas grey dots depict the $S_{\text {min }}$ patches. The two types are slightly shifted for better visibility.

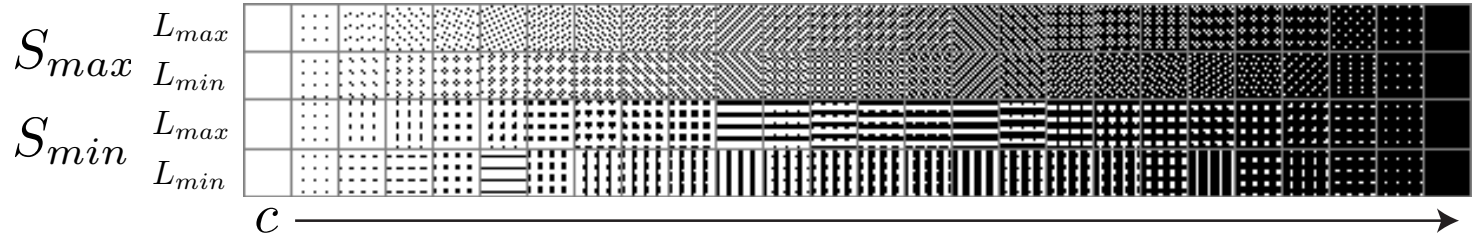

Figure 3. Patterns resulting in minimal and maximal luminance 


\section{CLUSTER MODEL}

In Ulichney, ${ }^{9}$ a simple model for cluster-finding is proposed based on a two-dimensional uncorrelated Gaussian filter $g$ with normal distribution $\Phi_{\mu, \sigma^{2}}(u, v)$. As the cells here repeat after $k$ pixels, a filter of infinite size is impractical. According to the theorem of Moivre-Laplace, the binomial distribution approximates the normal distribution $\Phi_{\mu, \sigma^{2}}$ with $\mu=n p$ and $\sigma^{2}=n p(1-p)$. We therefore define a two-dimensional binomial filter with $n=k-1$ and $p=1 / 2$ :

$$
f(u, v)=\left(\begin{array}{l}
n \\
u
\end{array}\right) p^{n}\left(\begin{array}{l}
n \\
v
\end{array}\right) p^{n} \quad u \in\{0 \ldots k-1\}, v \in\{0 \ldots k-1\}
$$

For every position $(x, y)$ in the cell, the binomial filter $f$ is convolved with the repetitive binary mask $M$ where a black pixel is defined as 1 and a white pixel as 0 :

$$
P(x, y)=\sum_{q=0}^{k-1} \sum_{p=0}^{k-1} M\left(x^{\prime}, y^{\prime}\right) f(p, q)
$$

where

$$
\begin{aligned}
& x^{\prime}=(\lceil k / 2\rceil+x+p) \text { modulo } k \\
& y^{\prime}=(\lceil k / 2\rceil+y+q) \text { modulo } k
\end{aligned}
$$

$P(x, y)$ defines, how clustered the surrounding of every pixel $(x, y)$ is. Equivalent to Ulichney, the largest cluster can therefore be identified using the maximum over all $P(x, y)$ whereas the minimum depicts the largest void. The difference between the maximum and the minimum describes the relative amount of clusters formed by the cell, which we call graininess :

$$
G=\max _{x, y} P(x, y)-\min _{x, y} P(x, y)
$$

As the pattern is repetitive, it is sufficient to search for the maximum and minimum within $0 \leq x, y \leq k-1$. Note that $G$ will be 0 both for the completely white and black pattern. Every other pixel arrangement will return a value for $G$ greater than 0 . We state that the value of $\min _{x, y} P(x, y)$ converges to 0 and the value of $\max _{x, y} P(x, y)$ converges to 1 with increasing $n$. As we observe dotgain effects to occur mostly with patterns of low graininess $G$, we further define the entropy of a pattern:

$$
E=1-G
$$

In this work, we were looking at patterns with $k=5$ defining a cell with 25 pixels. When, for example, we compute the entropy value for all distinct patterns with 5 black pixels out of 25 , we get a clear correspondance as

shown in figure 4, although further improvement is obviously possible. According to the observations in section 3, we use the entropy $E$ and the surface $S$ to define three types of patterns:

a) Patterns with minimal $E$ : AM-like patterns

b) Patterns with minimal $E$ whereas $S$ must be maximal: FM-like clustered patterns

c) Patterns with maximal $E$ whereas $S$ must be maximal: FM-like unclustered patterns

Using exhaustive search, multiple possible patterns can be found for most ink coverages $c$, therefore we choose one representative for each pattern type and each $c$. The resulting patterns are shown in figure 5 .

The AM-like patterns define some kind of a round dot screen which differs from the observations made in section 3. But as stated above, we neglect differences between the patterns with minimal S, assuming the resulting luminances are similar. Nonetheless, we assume the directional dependency of the patterns to be the main reason for the remaining uncertainity in figure 4 . 


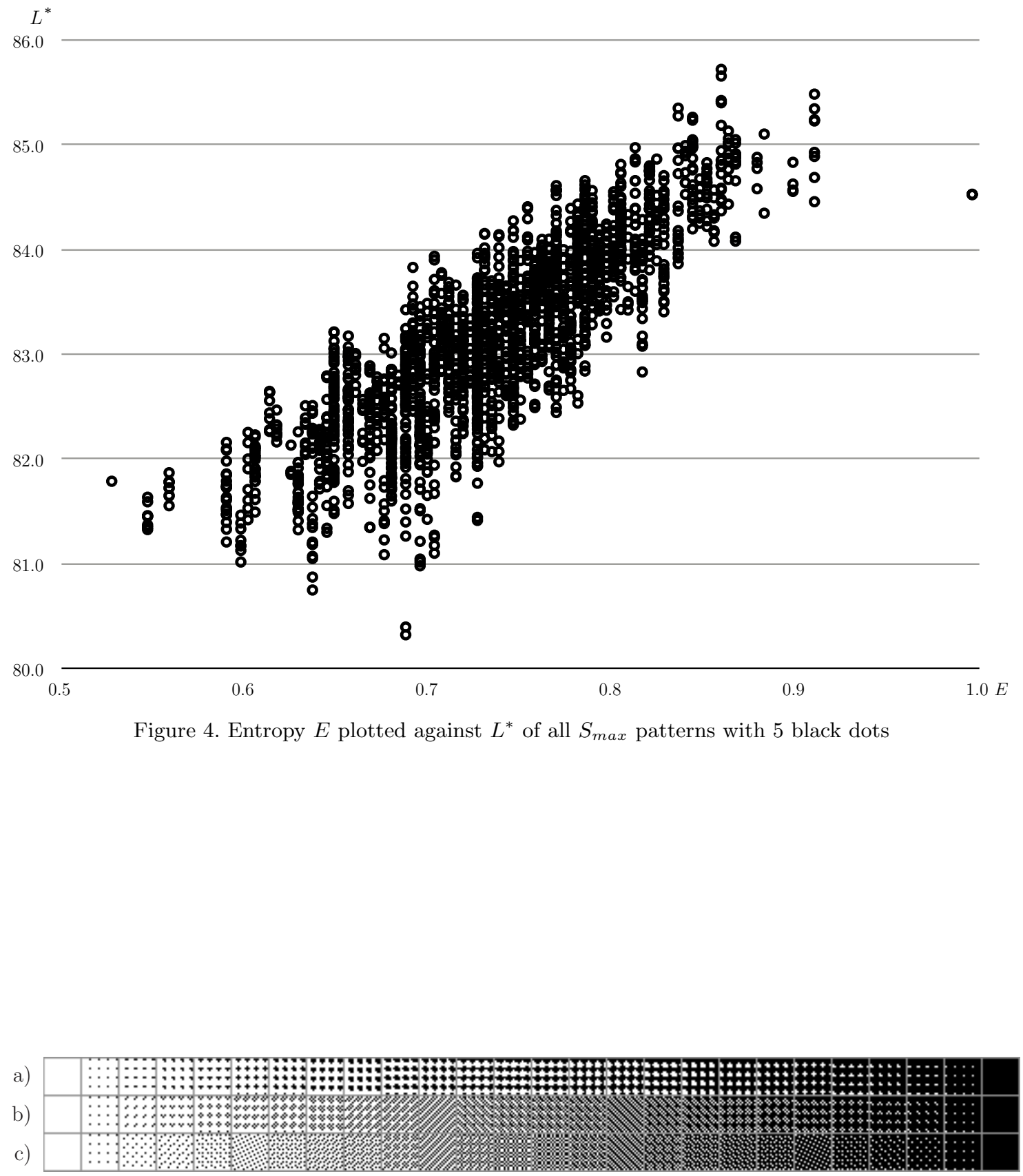

Figure 5. Three types of artificially created patterns: AM-like, FM-like clustered and FM-like unclustered. 


\section{DOTGAIN MODEL}

In the work of $\mathrm{Wang}^{2}$, repetitive $2 \times 2$ cells are proposed, therefore $2^{2 \times 2}=16$ possible dot arrangements of such a cell exist. As a simplification, the 16 cell types are grouped into 6 different types of patterns as shown in figure 6 , whereas the groups also contain the mirrored and rotated versions of the patterns. Each $2 \times 2$ cell defines a theoretical coverage $c_{0000}$ to $c_{1111}$.

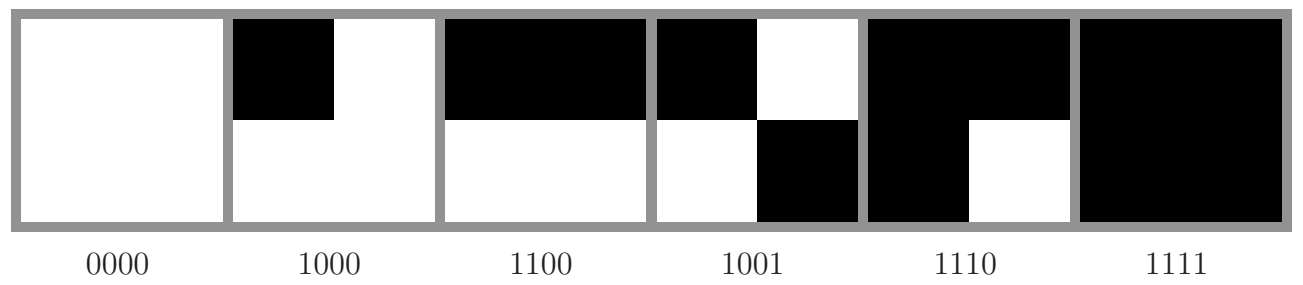

Figure 6 . the 6 groups of $2 \times 2$ patterns

These repetitive patterns are printed and measured using a densitometer resulting in luminance values $\ell_{0000}$ to $\ell_{1111}$. To predict the luminance of an arbitrary bitmap $M$, we create a decomposition of $M$ by counting the number of occurencies $w_{0000}$ to $w_{1111}$ of the $2 \times 2$ cells shown above within the bitmap. Note that due to cell overlap, pixels are accounted multiple times, we therefore normalize the system so that the sum of the weights $w$ equals 1 . Therefore, the luminance $L(M)$ of a given bitmap can be predicted using a linear combination of the parameters $\ell$ with the weights $w$. In the same way, the theoretical coverage $c$ can be decomposed into a 6 dimensional space.

$$
\begin{aligned}
& w_{0000} \cdot \ell_{0000}+w_{1000} \cdot \ell_{1000}+\cdots+w_{1111} \cdot \ell_{1111}=L(M) \\
& w_{0000} \cdot c_{0000}+w_{1000} \cdot c_{1000}+\cdots+w_{1111} \cdot c_{1111}=c(M)
\end{aligned}
$$

This method is fully described in the work of $\mathrm{Wang}^{2}$ and in the further text, we will refer to this prediction as "Method 1". Regarding our approach, the decomposition splits the theoretical coverage into independent parts. We therefore incorporate the entropy model discussed in section 4 by defining an entropy weighting vector e which is the multiplication of the weights $w$ with the entropy $E(M)$ of the bitmap. Written in vector form, this is defined by:

$$
\mathbf{e}(\mathrm{M})=\mathrm{E}(\mathrm{M}) \cdot \mathbf{w}(\mathrm{M})
$$

In our approach, according to section 4 , we examine the $5 \times 5$ cells shown in figure 5 and compute the weights $w_{0000}$ to $w_{1111}$ accordingly. We define 6 additional parameters $d_{0000}$ to $d_{1111}$ depicting the unknown entropy base vector. This results in our prediction model:

$$
\mathbf{w}(\mathrm{M}) \cdot \ell+\mathbf{e}(\mathrm{M}) \cdot \mathbf{d}=\mathrm{L}(\mathrm{M})
$$

By measuring the luminance values $L$ of the printed patterns shown in figure 5 , we define an over-determinated equation system which can be solved using linear least squares. We propose a "Method 2" which solves for the vector $\mathbf{d}$ using the following equation system:

$$
\mathbf{e}(\mathrm{M}) \cdot \mathbf{d}=\mathrm{L}(\mathrm{M})-\mathbf{w}(\mathrm{M}) \cdot \ell
$$

This results in 6 fitted correction parameters $d_{0000}$ to $d_{1111}$. In addition, we propose a "Method 3 " where we do not rely on the measured luminances $\ell$ but rather predict them the same way as the correction parameters $\mathbf{d}$ using linear regression by defining the following 12 dimensional equation system:

$$
\left(\begin{array}{l|l}
\mathbf{w}(\mathrm{M}) & \mathbf{e}(\mathrm{M})
\end{array}\right)\left(\begin{array}{l}
\ell \\
\mathbf{d}
\end{array}\right)=\mathrm{L}(\mathrm{M})
$$




\section{RESULTS}

The measurements $\ell_{0000}$ to $\ell_{1111}$ in $L^{*}$ are shown in table 1 for two paper types: A high quality paper for laser printers and a cheap paper from a scratchpad.

\begin{tabular}{|r||r||r|}
\hline & quality paper & cheap paper \\
\hline$\ell_{0000}$ & 94.65 & 90.26 \\
$\ell_{1000}$ & 84.13 & 83.90 \\
$\ell_{1100}$ & 49.97 & 53.07 \\
$\ell_{1001}$ & 45.24 & 48.74 \\
$\ell_{1110}$ & 26.53 & 30.56 \\
$\ell_{1111}$ & 17.08 & 22.85 \\
\hline
\end{tabular}

Table 1. Measured $L^{*}$ values of the 6 patterns of figure 6 .

Printing and measuring the patterns shown in figure 5, we gathered the data for the overdeterminated equation system and solved for the required parameters using linear least squares resulting in the parameters shown in table 2 .

\begin{tabular}{|c||r|r||r|r|}
\hline \multicolumn{1}{|c||}{} & \multicolumn{2}{c||}{ quality paper } & \multicolumn{2}{c|}{ cheap paper } \\
& method 2 & method 3 & method 2 & method 3 \\
\hline$\ell_{0000}$ & & 95.6397 & & 95.1935 \\
$\ell_{1000}$ & & 99.1329 & & 95.9212 \\
$\ell_{1100}$ & & 73.2902 & & 67.1954 \\
$\ell_{1001}$ & & 44.1155 & & 54.7917 \\
$\ell_{1110}$ & & 18.3895 & & 22.9544 \\
$\ell_{1111}$ & & 10.5662 & & 16.3357 \\
$d_{0000}$ & 0.9771 & -1.3473 & 1.6137 & -4.4864 \\
$d_{1000}$ & 4.8126 & -14.5540 & 4.3184 & -10.2084 \\
$d_{1100}$ & 9.5159 & -30.8092 & 2.5446 & -18.6452 \\
$d_{1001}$ & -5.4806 & -3.9200 & -2.5770 & -8.4214 \\
$d_{1110}$ & -4.9684 & 0.2292 & -2.3318 & 5.8301 \\
$d_{1111}$ & -0.4913 & 4.0657 & -1.0046 & 6.6360 \\
\hline
\end{tabular}

Table 2. Fitted parameters for the proposed methods.

To show the differences in luminance prediction of the three presented methods, we printed and measured patches filled with random patterns distributed within $0 \leq c \leq 1$. Table 3 shows the minimal and maximal prediction deviation and the average and the standard deviation $\sigma$ of the errors of the measured testpatches.

\begin{tabular}{|l||r|r|r||r|r|r|}
\hline \multicolumn{1}{|c||}{} & \multicolumn{3}{c||}{ quality paper } & \multicolumn{3}{c|}{ cheap paper } \\
& method 1 & method 2 & method 3 & method 1 & method 2 & method 3 \\
\hline $\min \Delta L$ & -6.12 & -6.04 & -4.75 & -5.37 & -5.40 & -5.76 \\
\hline $\max \Delta L$ & 3.42 & 1.71 & 1.48 & 3.51 & 2.09 & 1.36 \\
\hline $\operatorname{avg} \Delta L$ & -1.26 & -2.03 & -1.82 & -1.03 & -1.58 & -1.67 \\
\hline$\sigma \Delta L$ & 2.85 & 1.89 & 1.39 & 2.68 & 1.88 & 1.47 \\
\hline
\end{tabular}

Table 3. Resulting lightness differences

The proposed methods in general show an improvement in the standard deviation of the luminance prediction. Nevertheless, the proposed method predicts the luminances too high. The reason for this lays in the incorporation of the measurements of the AM-like patterns which contribute to the linear fit in an exceeding way. As patches filled with random dots tend to bulid FM-like structures, our prediction model must be defined using a corresponding base. We therefore calibrate our proposed method 2 and 3 with the patches b) and c) of figure 5 and we get the results shown in table 4 . 


\begin{tabular}{|l||r|r|r||r|r|r|}
\hline \multicolumn{1}{|c||}{} & \multicolumn{3}{c||}{ quality paper } & \multicolumn{3}{c|}{ cheap paper } \\
& method 1 & method 2 FM & method 3 FM & method 1 & method 2 FM & method 3 FM \\
\hline $\min \Delta L$ & -6.12 & -3.42 & -2.44 & -5.37 & -4.63 & -2.10 \\
\hline $\max \Delta L$ & 3.42 & 2.78 & 5.58 & 3.51 & 2.63 & 9.18 \\
\hline $\operatorname{avg} \Delta L$ & -1.26 & -0.22 & 0.70 & -1.03 & -0.72 & 1.77 \\
\hline$\sigma \Delta L$ & 2.85 & 1.67 & 1.78 & 2.68 & 1.77 & 2.59 \\
\hline
\end{tabular}

Table 4. Resulting lightness differences with calibration using only FM-patches

This results for most cases in a much more accurate prediction. We state that further improvement is expected when using our methods in combination with error diffusion algorithms, as such algorithms by definition are an approximation of the entropy discussed in this work.

\section{CONCLUSION}

The decomposition of the luminance into a 6-dimensional space is a basic concept indicated by the work of Wang. We enhanced this concept by adding a deviation vector consisting of a graininess description resulting in more accurate dotgain predictions. We estimate the final luminance of a dot including a surrounding cell by measuring only a fraction of all possible dot arrangements. In the future, this number may even be decreased by reducing the amount of redundancy.

We expect this method to be applicable to different printing methods, especially to offset printing. In the future, more printers and printing methods with high resolution will be tested. A further improvement of the model would be to use a different model for the clusters and to incorporate the printer dependent directional dependency of the printed pattern, resulting in a more relevant deviation vector.

\section{REFERENCES}

[1] J. A. C. Yule, W. J. Nielsen, "The penetration of light into paper and its effect on halftone reproduction", 1951, TAGA Proceedings, p. 65-76

[2] S. Wang, "Novel Centering Method for Overlapping Correction in Halftoning", Proceedings of IS\&T, 47th Annual Conference, 1994, pp. 482-486

[3] F. Mayr, "Untersuchung der Tonerpartikeldichte in Abhängigkeit der Druckpunktumgebung", Diploma thesis, August 2006, Supervisors H. Schmidt, S. Mourad

[4] H. E. J. Neugebauer, "A Describing Function for the Modulation Transfer of Xerography", Applied Optics IP, vol. 4, Issue 4, p. 453

[5] K. Simon, "Farbe im Digitalen Publizieren", Springer 2008, ISBN 978-3-540-37327-8

[6] M. Sormaz, S. Mourad, T. Stamm, P. Jenny, "Predicting Spectral Halftone Measurements for Different Instruments Using a New Multi/Scale Approach", CGIV 2008

[7] H. R. Kang, "Digital Color Halftoning", SPIE / IEEE Series on Imaging Science \& Engineering, 1999, ISBN 0-8194-3348-7, Chapter 7

[8] Rene Rasmussen, Edul N. Dalal, Kristen Hoffman, "Measurement of Macro-uniformity: Streaks Bands Mottle and Chromatic Variations", PICS 2001: 90-95

[9] R. Ulichney, "The void-and-cluster method for dither array generation", Human Vision, Visual Processing, and Digital Display IV, J. Allebach and B. Rogowitz, eds., Proc. SPIE 1913, pp. 332-343, 1993.

[10] Karl Haller, "Einfluss der Rasterpunktform auf die Übertragungsvorgänge bei autotypischen Druckverfahren", FOGRA-Forschungsbericht 6.029 